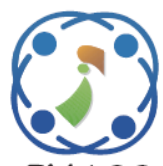

\title{
A Hybrid Face Image Contrast Enhancement Technique for Improved Face Recognition Accuracy
}

\author{
Shree Devi Ganesan $^{1 *} \quad$ Munir Ahamed Rabbani Mohammed ${ }^{1}$ \\ ${ }^{1}$ B.S. Abdur Rahman Crescent University, Tamil Nadu, India \\ * Corresponding author’s Email: shreedevi@bsauniv.ac.in
}

\begin{abstract}
Automatic Face recognition system struggles to recognize face images acquired at varying illumination conditions, facial expression, aging, and pose. The focus of this research work is to enhance the illumination affected face images, which subsequently results in improved face recognition accuracy. This paper presents a new contrast enhancement technique for face images. It is a hybrid contrast enhancement technique based on the combinatorial approach of Completely Overlapped Uniformly Decrementing Sub-block Histogram Equalization (COUDSHE) with various neighbourhood metric. COUDSHE concentrated to bring out a unique framework for localizing a global technique to adapt efficiently to local lightening conditions. The idea of using neighbourhood metric with histogram equalization results in enhancing the contrast of the image. Hence, the combination of COUDSHE with neighbourhood metrics results in enhanced images that are both adaptive to local brightness and good contrast enhancement. The application of our hybrid technique on the Extended Yale B Database has proved to improve the contrast of images and as a result, there is significant improvement in the recognition accuracy of the face recognition system implemented using Principal Component Analysis.
\end{abstract}

Keywords: Contrast enhancement, Localization, Neighbourhood metrics, Global histogram equalization, Fuzzy approach, Sub blocks histogram Equalization, Principal component analysis.

\section{Introduction}

Automatic Face Recognition system dominantly spreads its wings of application into surveillance, access control, and forensic investigations. Face remains to be the appealing biometric measure due to its potential for integration with any environment supported by installation of a digital camera. In 2009 , statistical reports showed that, to shoot 4 billion hours of footage a week, around 30 million surveillance cameras were used [1]. Face recognition has its applications in online face search and family photo-album organization [2]. However, recent advances in face recognition battles against the challenges in real time environment, computer vision is yet to duplicate the capabilities of human. Challenges posing face recognition systems are facial appearance variation due to expression, illumination, pose, aging, occlusions, disguise and so on [3]. Our work concentrates on the challenges posed by illumination variation in face recognition.

Images acquired in real time environment are subject to vary in intensity and direction of lightening source $[4,5]$. This causes the acquired image to have strong shadows and less clarity. Illumination changes causes huge variation among the facial images of an individual compared to the variation of the facial image of different individual $[3,5]$.

As an outcome of extensive research to address the challenges in illumination, many approaches have evolved. There are three categories in Illumination pre-processing approaches. They are namely gray level transformation, Gradient or edge extraction and Reflectance field estimation Techniques [3]. The concept behind gray level transformation technique is remapping the pixel intensity values using a transformation function. The concept behind gradient and edge extraction 
technique lies in the extraction of edges present in the face image, which are not sensitive to illumination variation. The concept behind reflectance field estimation lies in representing face image as a product of reflectance field estimation and illumination component.

Histogram equalization (HE), Logarithmic Transformation, and Gamma Intensity Correction approaches are approaches based on gray level transformation technique. By cross category comparison gray level transformation technique and reflectance field estimation approaches preserve more facial features [3]. Histogram equalization is the most prominently used contrast enhancement technique due to its efficient performance on almost all type of images $[6,7,8]$. Histogram equalization is simple technique, which distributes pixel values uniformly to obtain a high contrast image [8].

The two categories of Histogram equalization technique are Local Histogram Equalization and Global Histogram Equalization techniques [9]. GHE is a holistic approach, which uses the full input image to obtain the gray level transformation function [6]. This results in a overall contrast improvement but GHE does not acclimatize to the local brightness. LHE overcomes the drawbacks of GHE. LHE uses a sliding window method to remap the intensities of each centre pixel in the window using the gray level transformation function obtained from the neighbourhood pixels $[6,10]$. Despite being computationally, high LHE results in over enhanced image $[6,10,11]$. To reduce the computation complexity and obtain the advantages of local adaptability Partially Overlapped Sub-Block Histogram Equalization (POSHE) was proposed [10]. POSHE is the sibling of LHE but more effective, faster, and computationally lower than LHE [10]. POSHE gives large contrast enhancements, which global histogram equalization methods cannot achieve, and proves to be simpler than local histogram equalization [10].

The concept behind completely overlapped uniformly decrementing histogram equalization is to propose a framework to localize the global technique, enhance the image efficiently by adapting to the local brightness and to reduce the computational complexity associated to LHE [11]. Completely overlapped uniformly decrementing histogram equalization (COUDSHE) framework reduces the computational complexity of LHE as in POSHE but overcomes the drawbacks of POSHE which suffers from equalizing the symmetrical image regions [11]. The principle behind the introduction of neighbourhood metrics with histogram equalization is to reduce the gaps between bins and to increase the spread of histograms [12, 13]. Image contrast is an important feature of image enhancement [12, 13]. Distinction Metric, Voting Metric, Average metric contrast difference metric were proposed to decompose the large bins into sub bins which are then equalized independently [13]. The result of equalizing the sub bins independently helps to remap the gray levels in same bin to different bins, which results in much flatter histograms. The theory behind Fuzzy approach on the neighbourhood pixels is to overcome the identified shortcomings of other neighbourhood metrics [14].

We have proposed a hybrid approach of using neighbourhood metrics in the COUDSHE framework. This hybrid approach provides solution for problems namely local adaptability of the global technique and increase in contrast of an image using neighbourhood metrics. The main idea behind COUDSHE framework is to achieve similar enhancements in the symmetric regions of the face. This idea is unique and it strongly contributes towards improved face recognition accuracy.

The organization of the rest of the paper is as follows. In Section 2 and 3, we present the related work, which discusses about various gray level illumination pre-processing techniques. In Section 4, presents the experimental results and discussions about the effectiveness of the proposed technique. Section 5 concludes this paper and Section 6 finally presents the future enhancements.

\section{Related work}

\subsection{Global histogram equalization (GHE)}

The goal of GHE is to increase the overall contrast of an image. Global Histogram Equalization stretches the dynamic range of the image histogram using the following transformation function [12, 13]:

$$
T(I)=I_{o}+\left(I_{(L-1)}-I_{i}\right) P_{i}
$$

Where, $\mathrm{P}_{\mathrm{i}}$ denotes the cumulative density function defined by

$$
P_{i}=\sum_{i=0}^{L-1} p_{i}
$$

$p_{i}$ denotes the probability of the gray level of any given pixel $\mathrm{i}$. 


$$
p_{i}=\frac{n_{i}}{N}
$$

$\mathrm{n}_{\mathrm{i}}$ denotes number of occurrences of $\mathrm{i}^{\text {th }}$ intensity in the image, $\mathrm{N}$ denotes the total number of pixels of any image, $\mathrm{I}_{0}$ and $\mathrm{I}_{\mathrm{L}-1}$ represents lowest and highest gray level value within which GHE aims to stretch the gray level values of the given image.

\subsection{Partially overlapped sub block histogram equalization (POSHE)}

In Partially Overlapped Sub block, Histogram Equalization (POSHE) a mask (sub block) of size equal to one-fourth of the image's size would ideal for consideration. Perform Histogram Equalization for that block and then the mask is moved (stepped) half the size of the mask defined. Now the mask partially overlaps the sub block initially considered and new portion of the image. Perform histogram equalization for all the pixels in new sub block [10]. Usually the step size is half the size of the sub block defined. Fig. 1 shows the Regions bifurcation used in POSHE. Fig. 1 helps us to understand that the first sub block considered is R1, R2, R4, and R5. Perform histogram equalization is for the fore mentioned sub block. When the mask moves it moves only half the size of the sub block previously considered. Now, the regions under consideration are R2, R3, R5, R6, next proceed with application of Histogram Equalization for that block. Once there is no room for horizontal movement, the mask moves half the step size in the vertical fashion. Now, Regions R4, R5, R7, R8 undergoes Histogram Equalization. This process continues until the mask traverses the complete image. From Fig.1, it is clear that Region R1, R2, R4, R5 first undergoes histogram equalization. Then the mask shifts and Region R2, R5, R3, R6 undergoes histogram equalization. Average Pixel value will be the final value for the Regions that undergo histogram equalization more than once. Blocking Effect Reduction Filtering (BERF) eliminates the effects of blocks present in the illumination pre-processed image using POSHE.

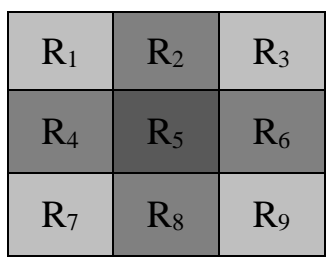

Figure.1 Framework for partially overlapped sub block histogram equalization
One of the drawbacks of POSHE is, when applying Histogram Equalization on the first block R1, R2, R3 and R4, normalization happens between these four regions. Partially overlapping the first block with the second block normalizes regions R2, R3, R5 and R6. Normalization of R1, R4, R3 and R6 with respect to each other does not happen. For facial images, these regions are symmetric region. Normalization of these regions becomes a mandatory condition. COUDSHE normalizes the face images considering the symmetric regions.

\subsection{Completely overlapped uniformly decrementing sub block histogram equalization (COUDSHE)}

The COUDSHE framework reduces the computational complexity of LHE as in POSHE but overcomes the drawback of POSHE, which suffers from equalizing the symmetrical image regions [11]. Fig.2 brings out the framework of COUDSHE. COUDSHE considers the whole image first and performs histogram equalization on the completely image. Uniformly decrementing all four sides of the image to obtain a new sub block. Perform histogram equalization on the sub block obtained. This process continues until the sub block size is minimal. Aggregate the pixel values that have undergone histogram equalization more than once. The illumination normalization obtained using COUDSHE has proved to outperform images preprocessed using POSHE [11].

\subsection{Neighborhood metrics (NM)}

The ideal greyscale image histogram is perfectly flat and makes use of every available grey value in the image format $[6,12]$. Application of GHE on illumination affected images, it is found that histograms do not use the entire range of gray scale value and the histograms are not flat [13]. Large histogram bins prominently exist and gap (empty bins) exists between large bins. The effect of such equalization results in low contrast images. Histogram equalization improves the overall contrast of the image but cease to improve the local

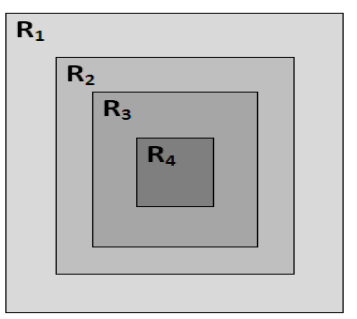

Figure.2 Framework for COUDSHE 
contrast on an image [14]. The intention of using neighbourhood metrics with histogram equalization is to use local information, to enhance the performance of global equalization by eliminating its drawbacks. Use of neighbourhood metrics with histogram equalization helps to map the image intensity value from the same bin to different bins, which increases the contrast of the image. Use of neighbourhood metrics reduces the gaps between the histograms and helps to achieve flatter histograms.

Face recognition accuracy improves as results of enhancing the facial regions affected by

blur and shadow. Neighbourhood metrics in use are Voting metric [6, 12, 13, 14], Distinction metric [6, $12,13,14]$, fuzzy approach on the neighbourhood pixels [14].

\subsubsection{Voting metric (VM)}

Voting metric considers the centre pixel and its eight neighbourhood pixels $[12,13]$. Voting metric counts the number of pixels lesser than the centre pixel among the eight neighbours in the $3 \times 3$ mask considered. Voting metric defined as $\beta_{m}(x, y)$.

$$
\beta_{m}(x, y)=\sum v\left(x, y, x^{\prime}, y^{\prime}\right)
$$

where

$$
\left(x^{\prime}, y^{\prime}\right) \in R_{m}(x, y)
$$

$R_{m}(x, y)$ represents region covered by the $3 \times 3$ mask.

$$
v\left(x, y, x^{\prime}, y^{\prime}\right)=\left\{\begin{array}{l}
1, c(x, y)>n\left(x^{\prime}, y^{\prime}\right) \\
0, \text { otherwise }
\end{array}\right.
$$

Fig. 3 presents the schematic diagram for evaluating the voting metric.

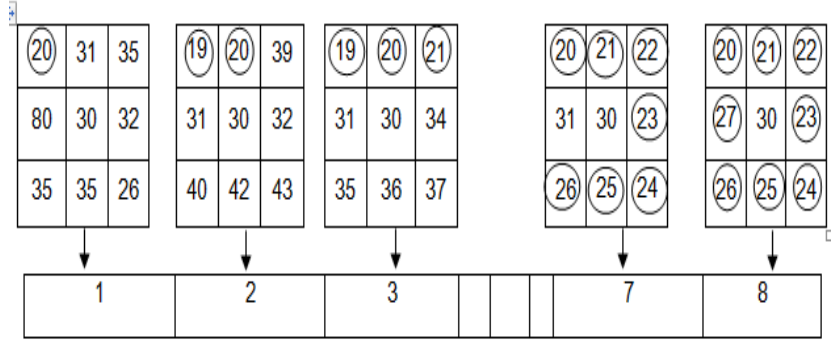

Figure.3 Illustration of voting metric

\subsubsection{Histogram equalization using voting metric}

Voting metric divides histogram bins into sub bins. Consider intensity value 30 and let its number of appearances be 300 times, and then voting metrics divides the bin, which represents the number of appearances into 8 sub bins. For each intensity value 30, its eight neighbourhood pixels are considered and among 8 neighbourhood pixels, pixels with intensity value less than 30 is counted. This represents voting count for an intensity value (here 30). The voting count for each occurrence of intensity value 30 is calculated. The centre pixel whose intensity value 30 and with same voting count are put in the same sub bin. After sub dividing the bins using voting metric, proceed with histogram equalization. Now, histogram equalization maps each sub bin of histogram 30 to different intensity values.

\subsubsection{Distinction metric (DM)}

The Distinction metric defined for a pixel $(\mathrm{x}, \mathrm{y})$ represented as $d_{m}(x, y)[6,13]$.

$$
d_{m}(x, y)=\sum t\left(x, y, x^{\prime} y^{\prime}\right)
$$

Where

$$
\left(x^{\prime}, y^{\prime}\right) \in R_{m}(x, y)
$$

$R_{m}(x, y)$ represents region covered by the $3 \times 3$ mask.

Distinction metric is summation of the difference between centre pixel and its neighbour for which centre pixel is greater than the neighbour pixel.

$$
t\left(x, y, x^{\prime}, y^{\prime}\right)=\left\{\begin{array}{l}
c(x, y)-n\left(x^{\prime}, y^{\prime}\right), c(x, y)>n\left(x^{\prime}, y^{\prime}\right) \\
0, \text { otherwise }
\end{array}\right.
$$

$c(x, y)$ is the centre pixel. $n\left(x^{\prime}, y^{\prime}\right)$ is the neighbourhood pixel within the $3 \times 3$ mask.

Fig.4 presents the schematic diagram for evaluating the distinction metric.

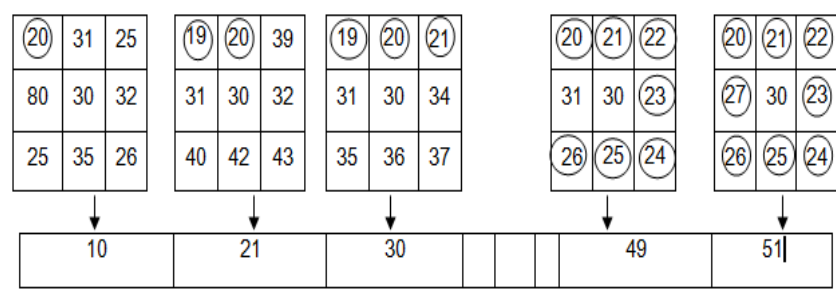

Figure.4 Illustration of distinction metric 


\subsubsection{Histogram equalization using distinction metric}

For each intensity value 30 , its eight neighbourhood pixels are considered and among 8 neighbourhood pixels, pixels with intensity value less than 30 is identified and their difference is measured and accumulated to find the distinction metric. Next, Group the intensity value with similar distinction metric into sub bin. Distinction metric divides each bin to a maximum of its (intensity value -0$)^{*} 8$ sub bins. After sub dividing histogram using distinction metric, perform histogram equalization. Now, histogram equalization maps each sub bin of histogram 30 to different intensity values.

\subsubsection{Fuzzy approach (FA) on the neighborhood pixels}

Voting and distinction metric uses the concept of crisp sets [14]. Voting and distinction metric sub divides larges bins by considering only the neighbourhood pixels less than the centre pixel. They discard the neighbourhood pixels greater than the centre pixel. The concept in fuzzy approach on the neighbourhood pixels is to consider all the eight neighbours of the centre pixel. The idea of considering all the eight neighbours is to characterise the centre pixel using all the eight neighbours. This leads to meaning full grouping of pixels in the same histogram. Fig.5 shows the schematic diagram of considering eight neighbours to cluster the centre pixel.

Fuzzy membership function estimates the relationship of each neighbouring pixels to the centre pixel. Same intensity value characterised with eight different neighbours are clustered using fuzzy - c means. Now, histogram equalization maps each cluster of histogram to different intensity values. The use of Fuzzy approach on neighbourhood pixels results in a meaning full grouping. The reason behind meaning full mapping is consideration of all the eight neighbours. The remapping capacity is maximum equal to the number of occurrences of pixels in the input image.
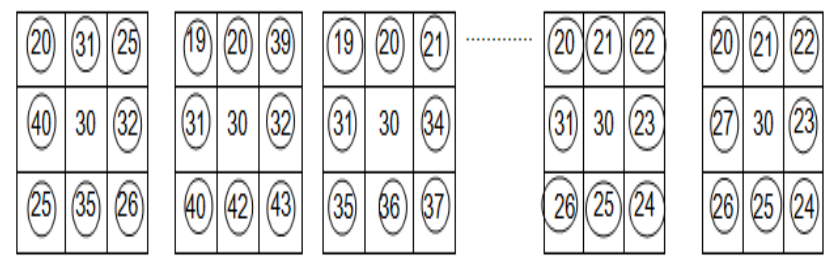

Figure.5 Illustration of fuzzy approach over the neighbourhood metric

\section{Proposed methodology}

Proposed methodology brings out a hybrid approach, which is the combination of COUDSHE and various the neighbourhood metric. The contribution of this approach has two main perspectives: firstly, to localize a holistic approach and enhance the image efficiently by adapting to the local brightness considering symmetrically regions for facial database using COUDSHE and secondly to improve the contrast of the image using neighbourhood metrics.

COUDSHE framework localizes the application of the global technique, by adapting to the local brightness of the image. It localizes the global technique by consider the symmetric regions for normalization. Symmetric regions of the face image contain important facial features that are essential for face recognition.

Histogram equalization with neighbourhood metric increases the spread of the histogram. Increase in spread results in contrast enhanced image. The facial features of a contrast enhanced face image are clearly distinguishable and subsequently improve the face recognition accuracy.

Contrast enhanced face images helps to distinguish the face features that are essential for recognition.

Procedure for COUDSHE with voting metric:

Step 1: Consider the full-face image.

Step 2: Define an output image array equal to the size of input image and set all the value to be zero.

Step 3: In the image for each occurrence of a given intensity value (say 10) consider the eight neighbours, find the vote of neighbours lesser than the centre pixel(say 10)

Step 4: Now, determine the sub groups for intensity value 10 using voting metric as the similarity measure.

Step 5: Repeat step 3 to step 4 for all the intensity values in the image.

Step 6: Perform Histogram Equalization and accumulate the results in temporary image array in the corresponding locations.

Step 7: $\quad$ Reduce the size of the block uniformly on all four sides.

Step 8: $\quad$ Repeat step 3 to 7 until the block size is minimal.

Step 9: Aggregate the intensity value in the corresponding location of the temporary array and store the result in the output 
image array.

Procedure for COUDSHE with distinction metric:

Step 1: $\quad$ Initially consider the full-face image.

Step 2: Define an output image array equal to the size of input image and set all the value to be zero.

Step 3: In the image for each occurrence of a given intensity value (say 10) consider all the 8 neighbourhood pixels., calculate the distinction metric by adding the difference between the centre pixel value and neighbouring pixels lesser than the centre pixel (say 10).

Step 4: Now, determine the sub groups for intensity value 10 using distinction metric as the similarity measure

Step 5: Repeat step 3 and step 4 for all the intensity values in the image.

Step 6: Perform Histogram Equalization and accumulate the results in temporary image array (same size as the input image) in the corresponding locations.

Step 7: Reduce the size of the block uniformly on all four sides.

Step 8: $\quad$ Repeat step 3 to 7 until the block size is minimal.

Step 9: Aggregate the intensity value in the corresponding location of the temporary array and store the results in the output image array.

Procedure for COUDSHE with fuzzy approach over the neighbourhood metrics:

Step 1: Consider the full image

Step 2: Define an output image array equal to the size of input image and set all the value to be zero.

Step 3: In the image for each occurrence of a given intensity value, apply triangular membership function to obtain the membership value of all the eight neighbours to the centre pixel. The membership value will range from $[0$, $1]$.

Step 4: $\quad$ Now apply fuzzy - c means clustering to create cluster using

the eight membership values as attributes. This helps to group pixels with intensity value 20 into different sub groups based on the 8 neighbourhood pixels

Step 5: $\quad$ Repeat step 3 and step 4 for all the pixel intensity in the image.
Step 6: After creating sub groups for each intensity value perform histogram equalization and accumulate the results in temporary image array in the corresponding locations.

Step 7: $\quad$ Reduce the size of the block uniformly on all four sides.

Step 8: Repeat step3 to step 7 until the block size is minimal.

Step 9: Now aggregate the intensity value in the corresponding location of the temporary array and store the result in the output image array.

\section{Experimental results and discussion}

Images considered for application of the proposed technique are present in Fig.5. These images are pre-processed using the COUDSHE framework with three different neighbourhood metrics namely the voting metric, distinction metric and the fuzzy approach over the neighbourhood metric. Fig. 6 shows the application of COUDSHE framework on illumination-affected images. Images pre-processed using proposed technique and resulting histogram representation are available in Fig.7. This helps to compare the performance of various neighbourhood metrics in the COUDSHE framework.

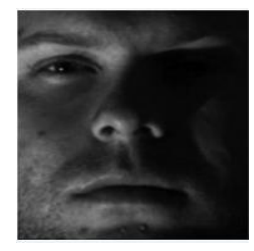

(a)

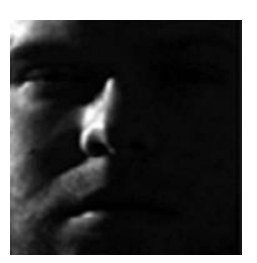

(b)

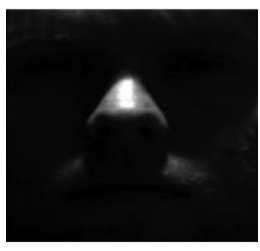

(c)
Figure.6 Illumination affected images considered for pre-processing: (a) image 1 is a heavily shadowed image, (b) image 2 is a half lit image, and (c) image 3 is a dark lit image

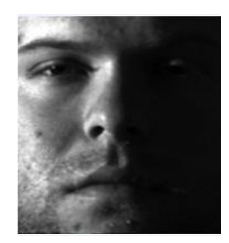

Region1

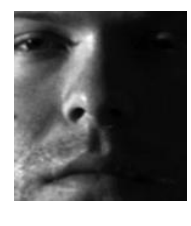

Region2
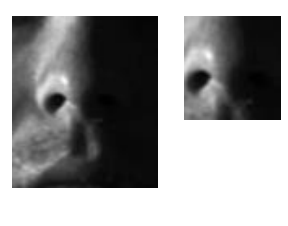

Figure.7 Application of COUDSHE framework on face image 

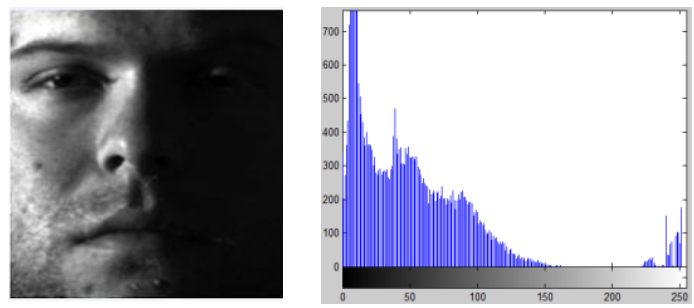

Image 1 and its Histogram representation
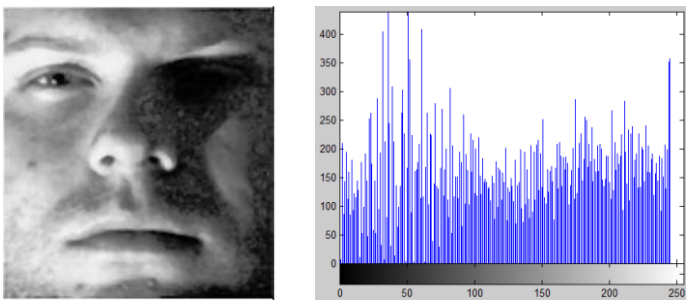

Image1 after Pre-processing with COUDSHE using VM and its histogram
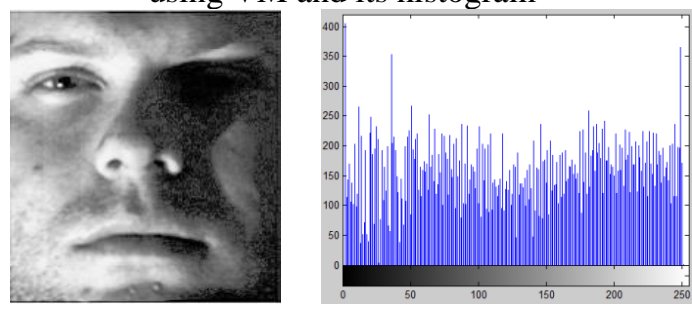

Image1 after Pre-processing with COUDSHE using DM and its histogram
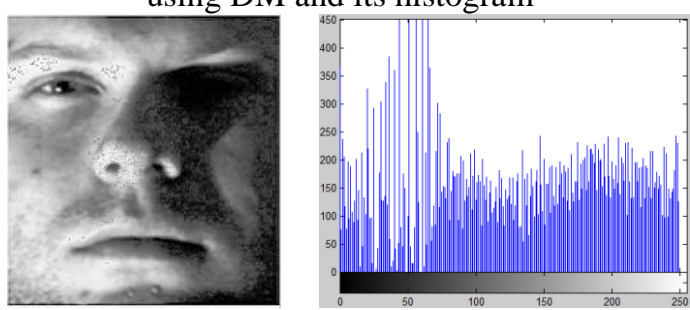

Image1 after Pre-processing with COUDSHE using FA over the NM and its histogram
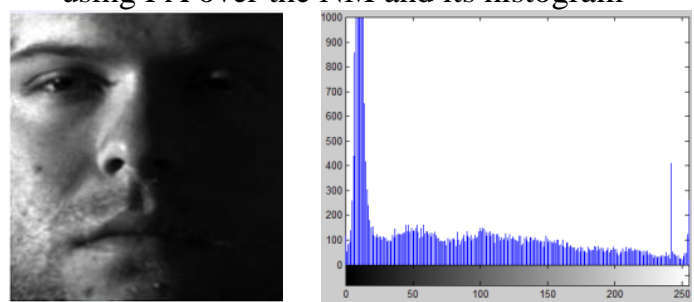

Image 2 and its histogram representation
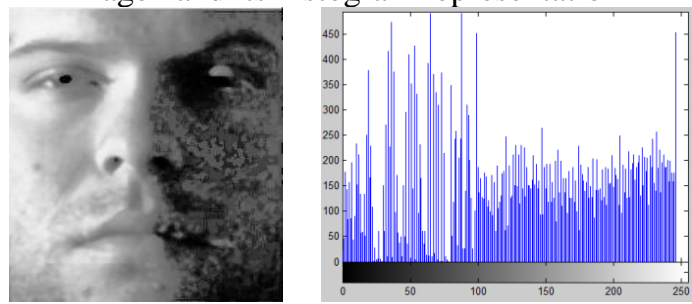

Image 2 after Pre-processing with COUDSHE using VM and its histogram
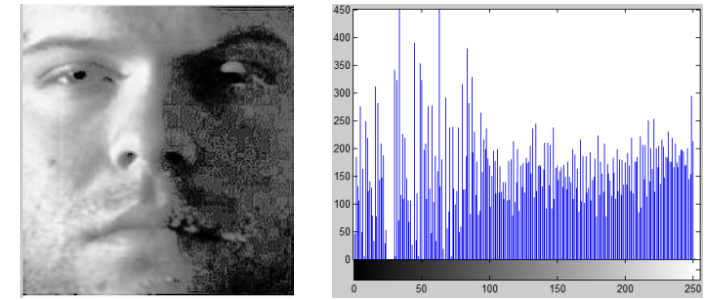

Image 2 after Pre-processing with COUDSHE using DM and its histogram
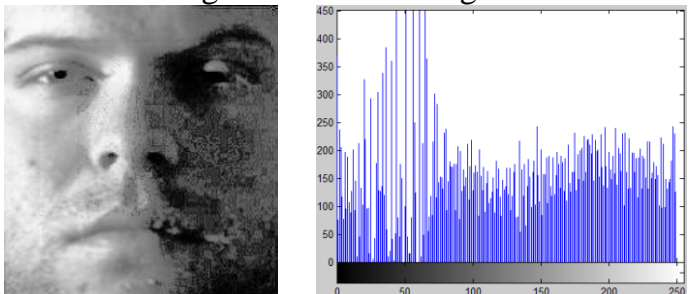

Image 2 after Pre-processing with COUDSHE using FA over the NM and its histogram
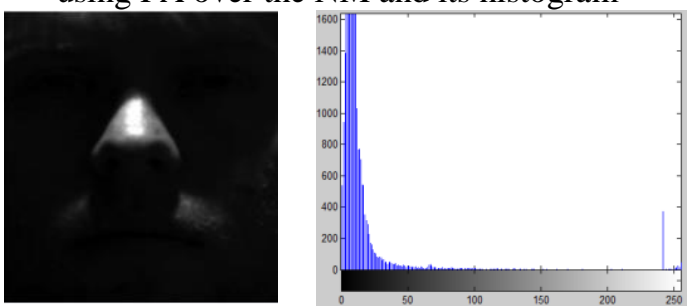

Image 3 and its histogram representation
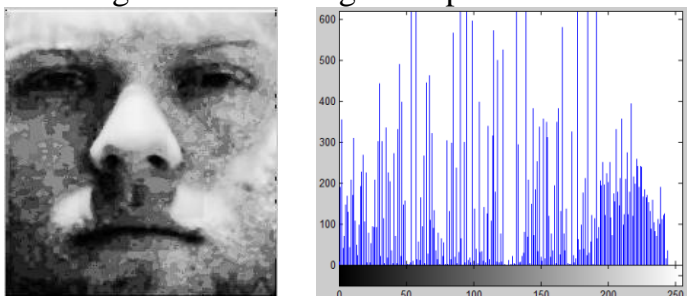

Image 3 after preprocessing with COUDSHE using VM and its histogram
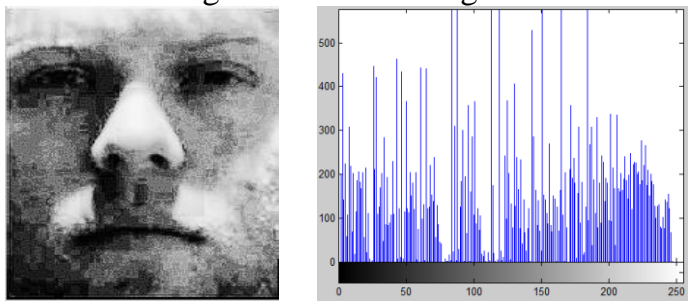

Image3 after preprocessing with COUDSHE using DM and its histogram
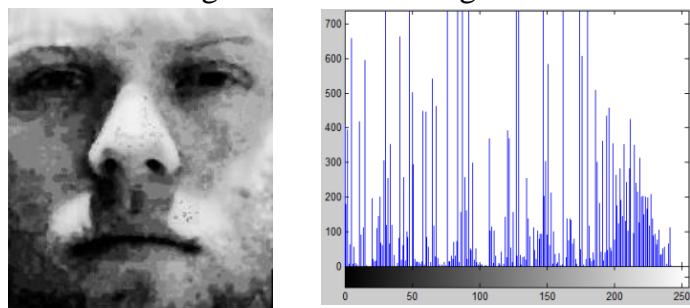

Image 3 after preprocessing with COUDSHE uisng FA over the NM and its histogram

Figure. 8 Resultant images after pre-processing.

[For all the histogram in Fig.8 the X-axis represent the intensity level in the image $[0,255]$ and Y-axis represents the frequency of occurrences] 
Table 1. Histogram flatness measure obtained from the sample images

\begin{tabular}{|c|c|c|c|c|c|c|}
\hline \multicolumn{7}{|c|}{ Histogram flatness measure $(\sigma)$} \\
\hline \multirow{2}{*}{ NM } & \multicolumn{2}{|c|}{ Heavily shadowed Image } & \multicolumn{2}{c|}{ Half lit image } & \multicolumn{2}{c|}{ Dark image } \\
\cline { 2 - 7 } & GHE & COUDSHE & GHE & COUDSHE & GHE & COUDSHE \\
\hline VM & 3846.7 & 2294.2 & 10100 & 9262.9 & 18879 & 9414.5 \\
\hline DM & 2879.7 & 2610.5 & 8907.6 & 7739.7 & 16323.2 & 7964.8 \\
\hline \multirow{2}{*}{ FA } & 2488.9 & 2478.3 & 9017.2 & 7587.6 & 17899.7 & 6896.6 \\
\hline
\end{tabular}

Table 2. Mean squared error obtained for sample images in Fig.5

\begin{tabular}{|c|c|c|c|c|c|c|}
\hline \multirow{2}{*}{ NM } & \multicolumn{4}{|c|}{ Mean Squared Error } \\
\cline { 2 - 7 } & \multicolumn{2}{|c|}{ Heavily shadowed Image } & \multicolumn{2}{c|}{ Half lit image } & \multicolumn{2}{c|}{ Dark image } \\
\hline VM & 6915.3 & 6847.0 & 6940.8 & 6688.8 & 6708.7 & 6938.8 \\
\hline DM & 7210.6 & 7059.2 & 7295.0 & 7234.0 & 7360.0 & 6679.5 \\
\hline FA & 6854.4 & 6993.9 & 6912.8 & 6555.5 & 6799.9 & 6489.3 \\
\hline
\end{tabular}

To evaluate the efficiency of the proposed technique the objective measures used to check the quality of the image are namely Histogram Flatness Measure (HFM) and Mean Squared Error. Histogram Flatness measure is used to measure the contrast of the image under consideration. HFM $(\sigma)$ of histograms is obtained from the following equation. Lesser Histogram Flatness measure indicates that Histograms are comparatively flatter.

$$
\sigma=\sum \frac{\left(\left|h_{i}\right|-\mu_{h}\right)^{2}}{D}
$$

where,

i varies between 0 to $D$

$\mathrm{D}$ is the range of intensity value.

|hi| is the size of the $\mathrm{i}^{\text {th }}$ bin of the image's histogram,

$\mu_{\mathrm{h}}$ is the mean size of the histogram bin.

Mean squared error (MSE) is defined to be the average difference between illumination enhanced image and image taken under proper illumination. Lesser the MSE value indicates closeness of the enhanced image in comparison with the image taken under good lightening condition. Compute MSE using the formula given in following equation.

$$
\mathrm{MSE}=\sum \frac{(\mathrm{X}-\mathrm{Y})^{2}}{\mathrm{MxN}}
$$

Where,

$\mathrm{X}$ is the illumination pre-processed image $\mathrm{Y}$ is the image taken under proper illumination condition.

$\mathrm{Mx} \mathrm{N}$ is the size of the given image.
Table 1 shows that the flatness measure $(\sigma)$ and Table 2 shows the MSE of the images pre-processed using GHE with various neighbourhood metrics and COUDSHE framework with various neighbourhood metrics. Lesser value of $\sigma$ indicates higher flatness ratio. Similarly Table 2 shows that there is decrease in MSE for the images pre-processed using COUDSHE framework with various neighbourhood metrics.

COUDSHE with voting metric performs well on shadowed images. The percentage of decrease in HFM is $7.4 \%$ on heavy shadowed images. COUDSHE with fuzzy approach over the neighbourhood metric performs well on half lit and dark lit images. Histogram flatness measure and Mean squared error records a lesser value compared to values obtained when pre-processing the images using existing methods. COUDSHE with fuzzy approach over the neighbourhood metric records $13.4 \%$ of decrease in HFM value for Dark lit images compared to other tabulated illumination enhancement techniques.

\subsection{Evaluation of face recognition accuracy using principal component analysis (PCA)}

COUDSHE frame work with Neighbourhood metrics has shown considerable decrease in the histogram flatness measure compared to the histogram flatness measure obtained for GHE using neighbourhood metrics. This implies that the contrast of the images has increased and hence the meaningfulness of the image has improved. COUDSHE with VM has the least MSE for heavy shadowed image and COUDSHE with fuzzy metric 
Table 3. Accuracy of the face recognition system using PCA

\begin{tabular}{|c|c|c|}
\hline Framework & Neighbourhood metric & Accuracy \% \\
\hline \multirow{7}{*}{ GHE } & Voting Metric & $67.6 \%$ \\
\cline { 2 - 3 } & Distinction Metric & $78.2 \%$ \\
\cline { 2 - 3 } & $\begin{array}{c}\text { Fuzzy Approach over the } \\
\text { Neighbourhood Metric }\end{array}$ & $81.34 \%$ \\
\hline COUDSHE & Voting Metric & $84.56 \%$ \\
\cline { 2 - 3 } & Distinction Metric & $87.02 \%$ \\
\cline { 2 - 3 } & $\begin{array}{c}\text { Fuzzy Approach over the } \\
\text { neighbourhood metric }\end{array}$ & $92.12 \%$ \\
\hline
\end{tabular}
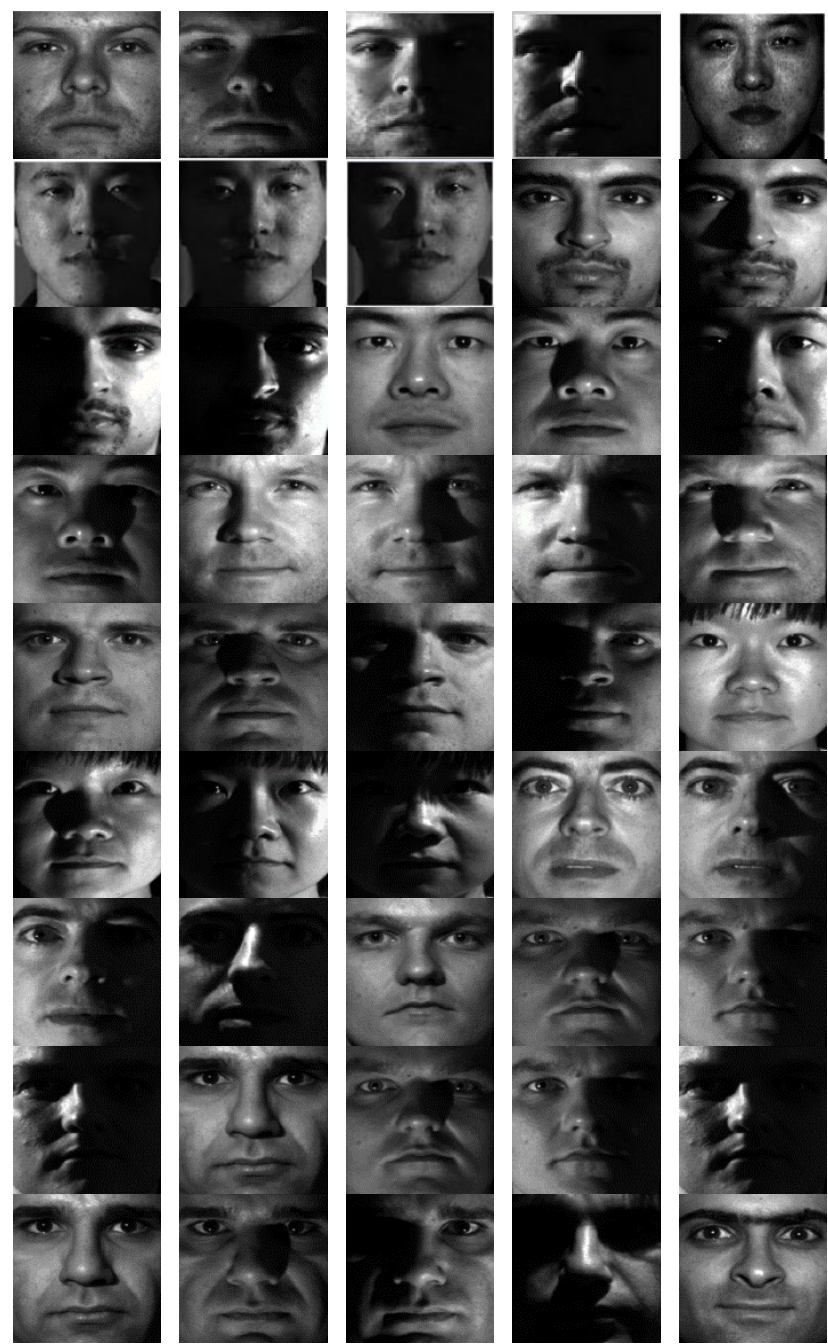

Figure.9 Set of face images shown as the training images

records good performance with reference to the histogram flatness measure and mean squared error for half-lit and dark lit images.

Now, we proceed to pre-process the images of 40 subjects taken under three different illuminations from Cropped Yale B Database. We have considered 120 images overall and pre-processed them using GHE using voting metric, GHE using distinction metric, GHE using Fuzzy approach, COUDSHE using voting metric, COUDSHE with

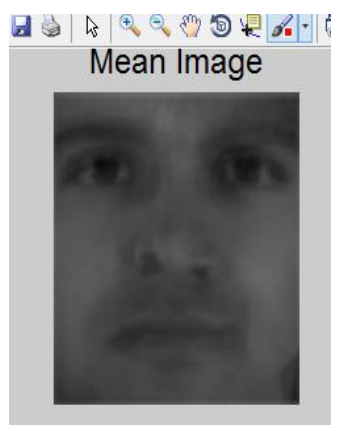

(a)

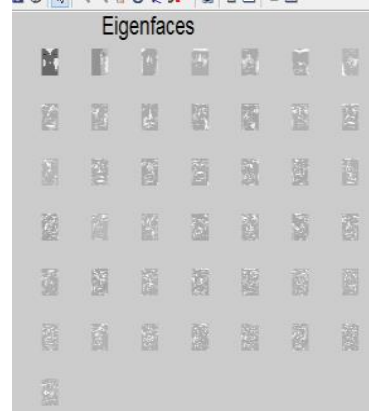

(b)

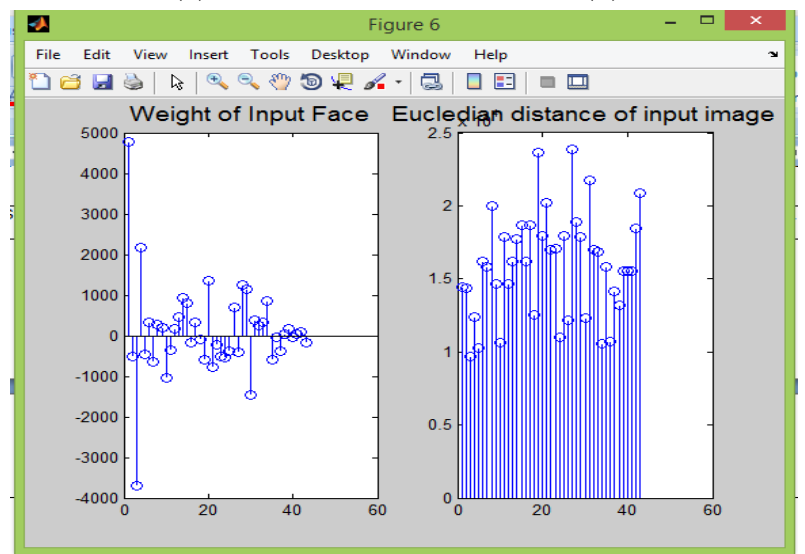

(c)

Figure.10 Intermediate results of PCA: (a) mean face image, (b) eigen face images, and (c) weight vector of the test image and Euclidean distance

Distinction Metric, CODUSHE with Fuzzy Approach over the neighbourhood metric. After preprocessing the face images affected by illumination variation, the next stage is to check whether there is increase in the recognition accuracy, which. One of the proven techniques for face recognition is Principal Component Analysis.

Principal Component Analysis Technique is a predictive analysis model and it reveals the internal structure of the data in a way which best explains the variance in the data. It is multivariate data analysis technique. Face image is $\mathrm{NxN}$ dimensional data, so it is $\mathrm{N}^{2}$ dimensional data. It transforms the higher dimensional data set into a low dimensional data.

Fig.9 shows the sub set of the training images considered from Cropped Yale B database. Fig.10 (a) shows the mean face image obtained for the training face images considered and Fig.10 (b) shows the eigen face images obtained for the training face images. Fig.10 (c) shows the weight vector graph and Euclidean distance between the weight vector of the test image and training images. PCA records improved recognition accuracy on contrast enhanced face images using our algorithm. The increase in the accuracy rate is $6 \%$. 


\section{Conclusion}

This paper brings out hybrid approach, which is a combination of Completely Overlapping Uniformly decrementing Sub block (COUDSHE) framework with various neighbourhood metrics. Experimental Results bring out the subjective and objective analysis of the proposed techniques. The objective analysis were evaluated and tabulated to determine the performance of the hybrid approach on facial images. Results reveal that the proposed technique adapts to the local brightness of the image and increases the overall contrast of the image. Histogram Flatness measure shows that COUDSHE with fuzzy approach almost outshines other combinations of COUDSHE with other neighbourhood approaches and GHE with various neighbourhood approaches for pre-processing image, which are dark lit and half-lit images. Experimental results also reveal that there is $6 \%$ increase in the recognition accuracy of the face images enhanced using the proposed technique. The proposed technique records good performance in terms of image contrast enhancement, and subsequently improves the recognition accuracy of the face images across varying illumination.

\section{Future enhancement}

COUDSHE brings out a novel framework for localizing the global technique. Fuzzy approach over the neighbourhood metric recommends the consideration of all the eight neighbourhood pixels for cluster the pixel with same intensity value. Using COUDSHE with fuzzy approach over the neighbourhood metrics for pre-processing videos captured in dark room for crime detection is the future enhancement of this work.

\section{References}

[1] A. K. Jain, B. Klare and U. Park, "Face Matching and Retrieval in Forensics Applications", IEEE Multimedia, Vol. 19, No. 1, pp. 20-28, 2012.

[2] A. Wagner, J. Wright, A. Ganesh, Z. Zhou, H. Mobahi, and Y. Ma, "Towards a Practical Face Recognition System: Robust Alignment and Illumination by Sparse Representation", IEEE Transactions on Pattern Analysis and Machine Intelligence, Vol. 34, No. 2, pp. 372-386, 2012.

[3] H. Han, S. Shan, X. Chen, and W. Gao, "A Comparative study on illumination pre processing in face recognition", Pattern Recognition, Vol. 46, No. 6, pp. 1691-1699, 2013.
[4] R. Gross, S. Bake, I. Matthews, and T. Kanade, "Face Recognition across Pose and Illumination", Handbook of Face Recognition Springer London, pp. 197-221, 2011.

[5] J.Y. Zhu, W.S. Zheng, F. Lu, and J.H. Lai, "Illumination invariant single face image recognition under heterogeneous lighting condition”, Pattern Recognition Vol. 66, pp. 313-327, 2017.

[6] N. Sengee, A. Sengee, and H.K. Choi, "Image contrast Enhancement using bi-histogram equalization with neighborhood metrics", IEEE Transactions of Consumer Electronics, Vol. 56, No. 4, pp. 2727-2734, 2010.

[7] S. Hashemi and S. Kiani, "An image contrast enhancement method based on genetic algorithm”, Pattern Recognition Letters, Vol. 31, No. 13, pp. 1816-1824, 2010.

[8] P. Rajavel, "Image dependent Brightness preserving Histogram Equalization", IEEE Transactions on Consumer Electronics, Vol. 56, No. 2, pp. 756 - 763, 2010.

[9] C. Lee, C. Lee, and C.S. Kim, "Contrast Enhancement Based on Layered Difference Representation of 2D Histograms", IEEE Transactions on Image Processing, Vol. 22, No. 12, pp. 5372-5384, 2013.

[10] J.Y. Kim, L.S. Kim, and S.H. Hwang, "An Advanced Contrast Enhancement using partially overlapped sub-block histogram equalization", IEEE Transactions on Circuits and Systems for Video Technology, Vol. 11, No. 4, pp. 475-484, 2001.

[11] G.S. Devi and M.A. Rabbani, "Image Illumination Enhancement based on Sub-block Histogram Equalization Technique", International Arab Journal of Information Technology. Accepted for publication.

[12] M. Eramian and D. Mould, "Histogram Equalization using Neighbourhood Metrics", In: Proc. of the Second Canadian Conf. In Computer and Robot Vision, pp. 397- 404, 2005.

[13] G.S. Devi and M.A. Rabbani, "Performance evaluation of GHE using Neighbourhood Metrics over Facial Databases", International Journal of Tomography and Simulation, Vol. 29, No. 2, pp. 27-36, 2016.

[14] G.S. Devi and MA. Rabbani, "Image Contrast Enhancement using histogram Equalization with fuzzy approach on the neighbourhood metrics", In: Proc. International Conf. on Wireless Communications, Signal Processing and Networking, Chennai, pp. 774- 777, 2016. 